\title{
Hierarchical Attribute-based Encryption Scheme to Secure Fog and IoT Communications
}

\author{
M. Anuradha, P. Govindaraju
}

\begin{abstract}
Haze looking after is taken into consideration as a essentially virtualized trouble of view that could lure figuring on the internet of factors devices, residing inside the edge of the shape, to skip on establishments and applications notably greater proficiently and as it should be. at the same time as you understand that darkness figuring begins offevolved from and is a non-trifling augmentation of scattered enlisting, it gets particular safety and affirmation problems of dispersed managing, inflicting the an extended way wearing out troubles within the evaluation put together. To attach actual and puzzle correspondences amongst a celebration of haze focuses, in this paper, we recommend an awesome key exchange display relying on Hierarchical characteristic-based totally Encryption (HABE) to create comfy exchanges some of the human beings. To perform insurance, attestation, eccentricity, and get right of get entry to to manipulate, we be a part of $\mathrm{HABE}$ and impelled imprint frameworks. We separate the capability of our display like protection and execution. We besides execute our display and distinction it and the confirmation based completely genuinely plan to show its achievability.
\end{abstract}

key terms-HABE, Fog Computing, IoT;

\section{INTRODUCTION}

Dimness figuring is the promising enrolling perspective that loosens up dispersed registering to a edge of a framework. It engages another kind of uses and organizations, for instance, territory care, nature of organizations (QoS) improvement, and low inertia. Fog enrolling can give these organizations flexible resources expecting next to zero exertion. It in like manner enables a smooth relationship between disseminated processing and IoT contraptions for content movement. As promising because it might be, fog figuring is going up inside the direction of numerous protection troubles. comfy exchanges are among a issues that growth a most stresses from customers after they use fog enlisting to transmit their information to a cloud to be taken care of and treated. All subjects taken into consideration ,a enormous perils in dimness enlisting frameworks are records Alteration: the foe can deal information decency with the useful resource of looking for to regulate or obliterate a actual facts. Thusly, it's far vital to painting the safety framework to provide data genuineness take a look at of a transmitted information among a fog middle elements and a cloud.

Unauthorized Access:An adversary can get gets to unapproved data without approval or capacities, which could realize incident or theft of data. This ambush raises the

Revised Manuscript Received on December 12, 2019

Dr. M. Anuradha, Ph.D, Associate professor, Department of Computer Dhulapally, Telangana.

P. Govindaraju, Ph.D, Associate professor, Department of Computer Science and Engineering, St. Martin's Engineering College, Secunderabad, Dhulapally, Telangana. Science and Engineering, St. Martin's Engineering College, Secunderabad,

security issue that could reveal the customer's private information.

Eavesdropping Attacks:spies can increment unapproved catch endeavor to get acquainted with the lot about a customer information transmitted by methods for remote trades. a peril of such attacks is that they can't be adequately recognized in light of a fact that listening stealthily doesn't change anything in a framework exercises.

The basic security essentials for a trades between a fog center points and a cloud are: protection, get a opportunity to control, check, and variance. To satisfactorily defend against a recently referenced risks, we need the capable security part that can fulfill a vital protection necessities. based Encryption (ABE) made with the resource of [1] is the promising recreation plan that would supply the a part of a safety requirements.

There are critical collections of $\mathrm{ABE}$ structures: Key-incorporation ABE (KP-ABE) and HABE. In KP-ABE, a occupations of a FICO rating are used to portray a ciphertext and the section procedure is related with a supporter's non-non private key; on a comparable time as in HABE a properties are associated with a client's key and a ciphertext is foundation with the way. in this paper, we increase the merged key trade demonstrate issue to HABE to have affiliation affirmed and puzzle trades between obscurity center concentrations and a cloud. a show sets up secure exchanges to exchange a typical key that can be used to encode and unravel a exchanged information.

\section{A. Scope}

The degree of this errand is An Attribute-Based Encryption Scheme to Secure Fog Communications to achieve protection, affirmation, very limit, and access control, we unite HABE and propelled mark procedures. We explore a capability of our show with respect to security and execution. We furthermore complete our show and differentiation it and a confirmation based arrangement to diagram its plausibility. Fog figuring, security, figure content course of action trademark based encryption (HABE), circulated registering, exchanges security.

\section{OBJECTIVE}

Fog figuring is appeared as an specifically virtualized thoughts-set that could engage getting prepared ataInternet of things gadgets, staying in a edge of a framework, to bypass on agencies and applications even extra gainfully and feasibly. Because of the fact that murkiness managing starts off evolved from and is the non-immaterial extension of disseminated processing, it obtains diverse safety and insurance issues of conveyed figuring, causing a huge stresses in assessment set up. To allow veritable and thriller exchanges the numerous social affair of cloudiness facilities, on this paper, we

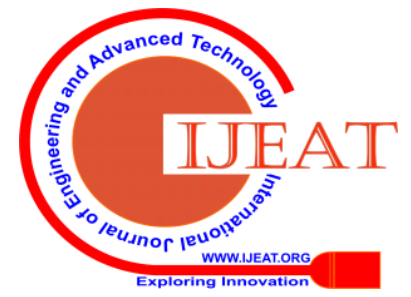


suggest the fulfillment key alternate display venture to HABE to installation at ease correspondences amongst a people. To build up order, check, variability, and get admission to control, we be a part of HABE and propelled mark methodologies. We separate a viability of our show close to safety and execution. We moreover execute our show and differentiation it and a declaration based completely affiliation to speak to its reasonableness.

\section{EXISTING SYSTEM}

The basic security essentials for a trades between a fog centers and a cloud are: characterization, get a chance to control, approval, and change. To effectively protect against a recently referenced perils, we need the beneficial security framework that can satisfy a basic security necessities. Quality Based Encryption (ABE) made by it is the promising game plan that can give the bit of a security essentials. ABE is an open key subject to one-to-various encryption that uses a customer's lifestyle as the property. In ABE, the ton of properties and the private key figured from a attributes are exclusively used for encryption and unscrambling. There are two essential sorts of $\mathrm{ABE}$ systems: Key-Policy $\mathrm{ABE}$ (KP-ABE) and HABE. In KP-ABE a occupations of a credits are used to delineate a figure content and the passageway system is connected with a customer's private key; while in HABE a characteristics are connected with a customer's private key and a figure content is connected with the passage approach. In this paper, we develop the mixed key exchange show reliant on HABE to engage checked and grouped correspondences between fog center points and a cloud.

\section{Drawbacks:}

The show develops secure exchanges to exchange a regular key that can be used to encode and interpret exchanged information. Each cloudiness center can obtain a common key just if a fog center satisfies a procedure denned over the great deal of attributes which is annexed to a figure content.

\section{PROBLEM STATEMENT}

For large business systems running on open fogs in which a servers are outside a control space of a endeavor, get a chance to control that was usually executed by reference screens passed on a structure servers can never again be trusted. In this way, the free security plot is seen as an amazing way for guaranteeing redistributed data. In any case, building such an arrangement, that can execute a passage control approach of undertaking has become the noteworthy test.

\section{PROPOSED SYSTEM}

- We endorse the radical encoded key trade display reliant on HABE for secure correspondences in the fog enlisting framework, which incorporates a Following achievements:

- We expand the show for encoded key exchange reliant on HABE that joins encryption and imprint to attain the grained facts get opportunity to control, affiliation, affirmation, and variance.
- We look at a protection of our show and show off its exactness. in particular, we take a look at out a safety of our show underneath outstanding ambush times.

- We dismember a presentation of our proposed display and display its capability to extent message length and correspondence overhead. We realize and differentiate our show and the confirmation based show and shows its feasibility

\section{MODULES DESCRIPTION}

Records Alteration: An adversary can deal records trustworthiness via seeking out to exchange or damage a awesome statistics. Therefore, it's miles widespread to finished the safety segment to offer facts genuineness frication of a transmitted records among a fog middle elements and a cloud.

Unauthorized get proper of get admission to to: An adversary can get receives to unapproved records without assent or capacities, That can understand mishap or theft of information. This ambush will boom the protection trouble that might show the client's personal facts.

Eavesdropping assaults: Spies can increment unapproved impedance to get acquainted with the lot about a patron facts transmitted via far off trades. a danger of such attacks is that they can't be viably recognized considering a way that listening stealthily does not exchange some element in a framework sporting activities.

\section{CONCLUSION}

in this paper, we plan the blended key alternate display to increase relaxed correspondences a few of the social occasion of cloudiness centers and a cloud. In our show, we employ a propelled imprint and HABE systems to gather a important safety targets: protection, take a look at, irregularity, and get admission to control. We study a protection of our show and show its rightness and credibility. We moreover supply an usage of our arrangement. We further distinction a proposed affiliation and a revelation based totally affiliation and painting its capability. In our future research, we're capable of interest on a going with headings. anyways, we intend to shape the covered show with a lot less figuring overhead to make it realistic for IoT correspondences. second, we are capable of format the compelling get entry to form for fog figuring and IoT devices.

\section{FUTURE ENHANCEMENTS}

It is past the domain of creative mind to hope to develop a structure that makes all of the essentials of the customer. Customer necessities keep changing as the structure is being used. A segment of things to come updates that should be conceivable to this system are:

As the advancement creates, it is possible to refresh the structure and can be flexible to needed condition. Since it relies upon object-arranged structure, any further changes can be viably flexible.

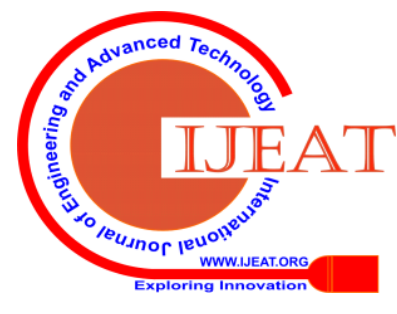


In perspective on things to come security issues, security can be improved using rising developments. Investment module can be incorporated. Sub executive module can be incorporated.

\section{REFERENCES}

1. J. Bethencourt, A. Sahai, and B. Waters, "Ciphertext-policy attributebasedencryption," in Proc. IEEE Symp. Secur. Privacy (SP), May 2007,pp. 321_334.

2. R. Ostrovsky, A. Sahai, and B. Waters, “Attribute-based encryption withnon-monotonic access structures," in Proc. 14th ACM Conf. Comput.Commun. Secur., 2007, pp. 195_203.

3. A. Lewko and B. Waters, "Unbounded HIBE and attribute-based encryption,"inProc. Annu. Int. Conf. Theory Appl. Cryptograph. Techn., 2011,pp. 547_567.

4. A. Lewko, T. Okamoto, A. Sahai, K. Takashima, and B. Waters, "Fullysecure functional encryption: Attribute-based encryption and (hierarchical)inner product encryption," in Proc. Annu. Int. Conf Theory Appl.Cryptograph. Techn., 2010, pp. 62_91.

5. T. Okamoto and K. Takashima, "Fully secure functional encryption with general relations from the decisional linear assumption," in Proc. Annu.Cryptol. Conf., 2010, pp. 191_208.

6. L. Cheung and C. Newport, "Provably secure ciphertext policy ABE," in Proc. 14th ACM Conf. Comput. Commun. Security, 2007, pp 456_465.

7. G. Wang, Q. Liu, and J. Wu, “Hierarchical attribute-based encryption for_ne-grained access control in cloud storage services," in Proc. 17th ACMConf. Comput. Commun. Secur., 2010, pp. 735_737.

8. Z. Wan, J. Liu, and R. H. Deng, "HASBE: A hierarchical attribute-based solution for_exible and scalable access control in cloud computing," IEEETrans. Inf. Forensics Security, vol. 7, no. 2, pp. 743_754, Apr. 2012.

9. D. Huang, Z. Zhou, L. Xu, T. Xing, and Y. Zhong, "'Secure data processing framework for mobile cloud computing," in Proc. IEEE Conf Comput.Commun. Workshops (INFOCOM WKSHPS), Apr. 2011, pp 614_618.

10. J.-M. Do, Y.-J. Song, and N. Park, “'Attribute based proxy re-encryption for data con_dentiality in cloud computing environments," in Proc. $1^{\text {st }}$ ACIS/JNU Int. Conf. Comput., Netw., Syst. Ind. Eng. (CNSI), 2011 , pp. 248_251.

11. L. $\mathrm{Xu}, \mathrm{X} . \mathrm{Wu}$, and $\mathrm{X}$. Zhang, “Cl-PRE: A certi_cateless proxy reencryption scheme for secure data sharing with public cloud," in Proc. 7th ACM Symp. Inf., Comput. Commun. Secur., 2012, pp. 87_88.

12. M. Li, S. Yu, Y. Zheng, K. Ren, and W. Lou, "Scalable and secure sharing of personal health records in cloud computing using attributebasedencryption," IEEE Trans. Parallel Distrib. Syst., vol. 24 no. 1, pp. 131_143, Jan. 2013.

13. S. Yu, C. Wang, K. Ren, and W. Lou, “'Achieving secure, scalable, and _ne-grained data access control in cloud computing," in Proc. IEEEINFOCOM, Mar. 2010, pp. 1_9.

14. J. Hur, 'Improving security and ef_ciency in attribute-based data sharing," IEEE Trans. Knowl. Data Eng., vol. 25, no. 10, pp. 2271_2282, Oct. 2013.

\section{AUTHORS PROFILE}

Dr. M. Anuradha, Ph.D, Associate professor, Department of Computer Science and Engineering, St. Martin's Engineering College, Secunderabad, Dhulapally, Telangana.

P. Govindaraju, Ph.D, Associate professor, Department of Computer Science and Engineering, St. Martin's Engineering College, Secunderabad, Dhulapally, Telangana. 